\title{
Few-cycle solitons in supercontinuum generation dynamics
}

\author{
Hervé Leblond ${ }^{1, a}$, Philippe Grelu ${ }^{2}$, Dumitru Mihalache ${ }^{3,4}$, and Houria Triki ${ }^{5}$ \\ 1 LUNAM Université, Université d'Angers, Laboratoire de Photonique d'Angers, EA 4464, \\ 2 Boulevard Lavoisier, F-49045 Angers Cedex 01, France \\ 2 Laboratoire Interdisciplinaire Carnot de Bourgogne, UMR 6303 CNRS, Université Bour- \\ gogne Franche-Comté, 9 avenue A. Savary, BP 47870, F-21078 Dijon Cedex, France \\ 3 Horia Hulubei National Institute for Physics and Nuclear Engineering, 30 Reactorului, \\ Magurele, 077125 Bucharest, Romania \\ 4 Academy of Romanian Scientists, 54 Splaiul Independentei, 050094 Bucharest, Romania \\ 5 Radiation Physics Laboratory, Department of Physics, Faculty of Sciences, Badji Mokhtar \\ University, P. O. Box 12, 23000 Annaba, Algeria
}

\begin{abstract}
We review several propagation models that do not rely on the slowly-varying-envelope approximation (SVEA), and can thus be considered as fundamental models addressing the formation and propagation of few-cycle pulsed field structures and solitary waves arising in the course of intense ultrashort optical pulse evolution in nonlinear media and beyond octave-bandwidth optical spectrum broadening. These generic models are: the modified-Korteweg-de-Vries $(\mathrm{mKdV})$, the sineGordon (sG), and the mixed mKdV-sG equations. To include wave polarization dynamics, the vector extensions of both $\mathrm{mKdV}$ and $\mathrm{sG}$ equations are introduced. Multi-octave-spanning supercontinuum generation and few-cycle soliton structures are highlighted from numerical simulations.
\end{abstract}

\section{Introduction}

At the turning of the last century, the improvement of titanium-sapphire laser cavities mode locked through the Kerr-lensing effect, including for instance chirped mirrors for precise dispersion compensation, allowed an efficient exploitation of the very large gain medium bandwidth with the direct generation of amazingly short laser pulses, with a duration down to a few optical cycles. As one of the first realizations, Ref. [1] reported in 2001 the generation of 5 fs-long pulses with a spectrum extending from 600 to $1200 \mathrm{~nm}$, namely, pulses about two optical cycles long spanning over an octave across the central 800-nm wavelength, directly from the laser oscillator. For such intense ultrashort pulses, even diluted media such as the surrounding air can manifest as strongly dispersive and nonlinear ones.

The question was then, how the propagation of such pulses could be modeled. Popular ultrashort propagation models had been centered around the slowly-varyingenvelope approximation (SVEA) that is fundamentally a perturbative approach valid

\footnotetext{
a e-mail: herve.leblond@univ-angers.fr
} 


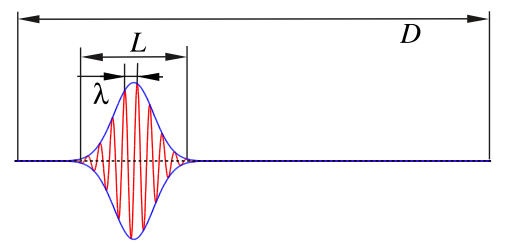

(a)

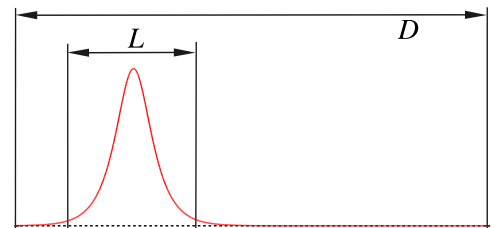

(b)

Fig. 1. Schematic representation of (a) envelope vs. (b) solitary wave types of solitons.

when the pulse duration is much longer than the optical cycle or, equivalently, when the spectral bandwidth is much smaller than the central frequency, which can thus be considered as a carrier frequency. At the lowest orders of dispersion and nonlinearity, propagation in cubic nonlinear media can be effectively described by the nonlinear Schrödinger (NLS) equation, which can be accurate for pulsewidths down to about ten optical cycles at moderate optical intensities. In addition, the NLS equation has served as a central model in the investigation of envelope soliton formation and propagation. With an increase in the optical intensity, additional nonlinear effects need to be included, such as Raman and self-steepening effects. As the optical bandwidth increases, higher-order dispersion terms cannot be neglected. Thus, within the frame of the SVEA, the answer to shorter and higher-intensity pulse modeling implies an increasing number of terms added to the NLS equation, which is the so-called generalized NLS equation (GNLSE) approach [2]. The GNLSE implies numerical integration, and has indeed provided successful modeling of supercontinuum generation experiments in well-characterized optical media such as silica glass fibers [3]. Despite its actual success in a range of applications for pulse duration down to a few optical cycles, the GNLSE relies on a large number of coefficients, and it is not integrable in general.

In the present contribution, our general motivation is to unveil stable few-cycle pulse propagation effects in the context of supercontinuum generation spanning over several octaves. This investigation, from the theoretical point of view, can hardly be undertaken in the frame of an arbitrarily high-order GNLSE since, beyond the mathematical complexity, the validity of the latter approach remains questionable. Instead, we propose to consider field models that do not use the SVEA, and rely on few equation parameters, allowing the investigation of general classes of few-cycle pulse dynamics.

At this point, let us emphasize on two main different types of solitons. This classification is decisive in the frame of the mathematical soliton theory of integrable equations and the inverse-scattering-transform method. One type corresponds to the envelope soliton, which is the usual description of optical solitons in the picosecond range (Fig. 1(a)). As discussed above, the definition of the envelope assumes the SVEA, which means that the pulse spatial extension $L$ is much larger than the wavelength $\lambda$. The other type, called a solitary wave, consists in a single oscillation, without any carrier wave (Fig. 1(b)). It is best portayed by the hydrodynamical soliton that was first observed by John Scott Russel in 1834 [4], and was modeled by the Korteweg-de Vries (KdV) nonlinear partial differential equation, which was in fact discovered by Boussinesq $[5,6]$. The case of few-cycle pulses (FCPs) may appear as an intermediate situation, where we could consider an envelope and a carrier. However, since the pulse duration $L$ has the same order of magnitude as the wavelength $\lambda$, the SVEA, which is intrinsically a perturbative approach, is not valid in general, and uselessly complicates the mathematical approach of solitary waves. Therefore a completely different approach to few-optical-cycle pulse propagation has been developed, 


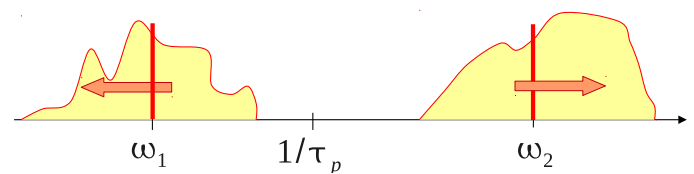

Fig. 2. General scheme of the material spectral model.

based on KdV-type models [7,8]; see also the reviews [9-11]. The first objective of this approach is to highlight a few classes of few-cycle pulse formation and solitary wave dynamics, which can be relevant in supercontinuum generation and other ultrashort pulse propagation in highly nonlinear waveguides. Note that in the following, the transverse spatial dimensions are excluded. At this early stage, since the parameters of the KdV-type equations are not easily connected with the experimental characterization parameters of materials, we leave a quantitative comparison between model predictions and experiments for future investigations, while focusing on the major physical mechanisms at play.

\section{Models for few-cycle solitons}

\subsection{Assumptions}

With the goal to build simple but effective models, some approximations were required, while taking into account the very large bandwidth required for FCP soliton propagation. The absorption spectrum of most transparent media contains two absorption bands in general: one below, and one above the transparency window. In a first approximation, we can reduce both absorption bands to single transitions as sketched in Fig. 2, so that the general material becomes modeled by the very simple model of a two-component medium, each component being described by density matrix equations for two-level models.

The approximation used to simplify the mathematical model is that the bandwidth of the transparency window is very large, i.e., we assume that the FCP duration $\tau_{p}$ is such that $\omega_{1} \ll\left(1 / \tau_{p}\right) \ll \omega_{2}$, where $\omega_{1}$ and $\omega_{2}$ are the central frequencies of the infrared (IR) and ultraviolet (UV) transitions, respectively.

\subsection{Models}

The $\mathbf{m K d V}$ equation. In a first stage, the two components are treated separately. We first consider the UV transition only, with the assumption that $\left(1 / \tau_{p}\right) \ll \omega_{2}$. This assumption corresponds to a long-wave approximation, which is performed according to the standard procedure of the reductive perturbation method, or multiscale expansion method [12]. It results in an asymptotic model, which is the modified Korteweg-de Vries (mKdV) equation, as [8]

$$
\frac{\partial E}{\partial \zeta}=\left.\frac{1}{6} \frac{d^{3} k}{d \omega^{3}}\right|_{\omega=0} \frac{\partial^{3} E}{\partial \tau^{3}}-\left.\frac{6 \pi}{n c} \chi^{(3)}(\omega ; \omega, \omega,-\omega)\right|_{\omega=0} \frac{\partial E^{3}}{\partial \tau} .
$$

Here $E$ is the electric field, $\zeta$ is the propagation distance and $\tau$ is the retarded time in a frame moving at the speed of light $c / n$, where $c$ is the light speed in vacuum, and $n$ is the medium optical index in the limit of long wavelengths and low amplitudes. The coefficients of Eq. (1) reflect in a simple general form the dispersion relation $k(\omega)$ 


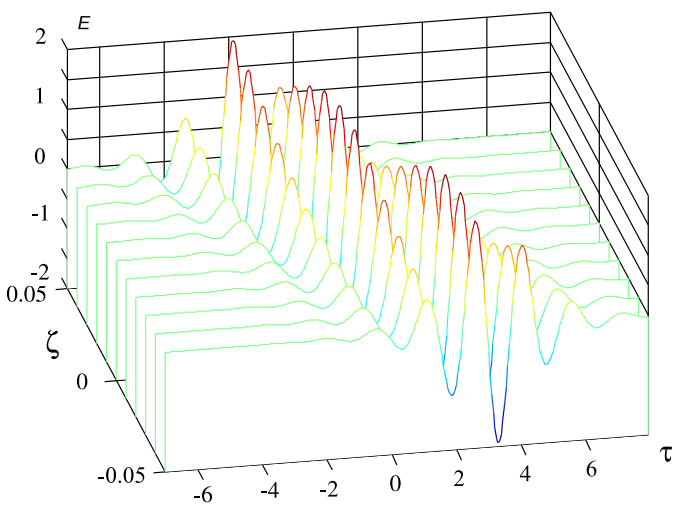

Fig. 3. The typical analytical breather solution.

of the medium and its cubic nonlinear susceptibility $\chi^{(3)}$. It should be emphasized that the $\mathrm{mKdV}$ equation is completely integrable by means of the inverse scattering transform (IST) method $[13,14]$.

The sine-Gordon equation. In a second stage, we consider the IR transition only, with the assumption $\omega_{1} \ll\left(1 / \tau_{p}\right)$. This corresponds to a short-wave approximation, which is another situation well-known within the frame of the reductive perturbation method, although a bit less standard than the previous one. It results in the sineGordon (sG) equation, as [8]

$$
\frac{\partial^{2} \psi}{\partial z \partial t}=c_{1} \sin \psi
$$

In Eq. (2), $c_{1}=w_{\infty} / w_{r}$ is the normalized initial population difference, and the field $\psi$ is defined so that $\partial \psi / \partial t=E / E_{r}$ is the normalized electric field. The propagation distance $z$ and retarded time $t$ are also normalized, see [8].

The $\mathbf{m K d V}$-sG equation. Then the two approximations are brought together to yield a general model, which is known as the $\mathrm{mKdV}$-sG equation. It is expressed in terms of the field $\psi$ as [15]:

$$
\frac{\partial^{2} \psi}{\partial z \partial t}+c_{1} \sin \psi+c_{2} \frac{\partial}{\partial t}\left(\frac{\partial \psi}{\partial t}\right)^{3}+c_{3} \frac{\partial^{4} \psi}{\partial t^{4}}=0,
$$

and in terms of the normalized electric field $u=\partial \psi / \partial t=E / E_{r}$ as

$$
\frac{\partial u}{\partial z}+c_{1} \sin \int^{t} u+c_{2} \frac{\partial u^{3}}{\partial t}+c_{3} \frac{\partial^{3} u}{\partial t^{3}}=0 .
$$

Equation (3) or (4) is integrable by the IST method in some special cases: when it reduces to the mKdV equation $\left(c_{1}=0\right)$, or to the sG equation $\left(c_{2}=c_{3}=0\right)$ [16], or if a specific relation between the coefficients is satisfied, namely $c_{3}=2 c_{2}$ [17]. In each integrable case it possesses an analytical solution, called a breather, which is a localized oscillatory pulse that propagates without deformation except for periodic oscillations (Fig. 3). The breather is hence a stable short pulse that propagates without being spread out by dispersion, and closely corresponds to the intended shape of a few-cycle optical soliton. 


\section{A few generalizations}

\subsection{General Hamiltonian}

We assumed that each of the IR and UV absorption bands could be reduced to a single transition. How does the models described above generalize to a higher number of atomic levels? In the case of the UV transitions, it has been shown that the mKdV model as given by Eq. (1) generalizes without modification to a general Hamiltonian, with an arbitrary number of atomic levels [18]. In the case of the IR transitions, the generalization is not so simple.

Indeed, the sG model (Eq. (2)) originates from the following coupled equations:

$$
\begin{aligned}
\frac{\partial E}{\partial z} & =\frac{-N}{\varepsilon_{0} c} \Omega Q, \\
\hbar \frac{\partial w}{\partial t} & =-E Q, \\
\hbar \frac{\partial Q}{\partial t} & =|\mu|^{2} E w,
\end{aligned}
$$

in which $\Omega$ is the angular frequency of the transition, $N$ is the density of atoms, $\mu$ is the dipolar momentum matrix element, $w$ is the population difference, and $Q$ is some auxiliary field, while obviously $\varepsilon_{0}$ is the dielectric permittivity of vacuum, and $\hbar$ is Planck's constant. The system (5)-(7) is formally identical to the equations of the self-induced transparency (SIT) equations, with the major difference that here $E$ and $Q$ are real fields, and $Q$ is not the polarization density, but is proportional to its $\tau$-derivative. We recall that the SIT model concerns the envelope of the field close to resonance, while Eqs. (5)-(7) concern the electric field itself, far from the resonance.

Since the population difference is explicitly involved, the generalization to a multilevel model requires one population difference $w_{j}$ variable for each transition. As an example, for a four-level system with two allowed transitions, the system (5)-(7) generalizes to [19]

$$
\begin{aligned}
\frac{\partial E}{\partial z} & =\frac{-N \Omega}{\varepsilon_{0} c}\left(\Omega_{1} Q_{1}+\Omega_{2} Q_{2}\right), \\
\hbar \frac{\partial w_{j}}{\partial t} & =-E Q_{j}, \quad j=1,2, \\
\hbar \frac{\partial Q_{j}}{\partial t} & =\left|\mu_{j}\right|^{2} E w_{j}, \quad j=1,2,
\end{aligned}
$$

which in turn reduces to

$$
\frac{\partial u}{\partial z}+c_{1} \sin \int^{\tau} u d \tau^{\prime}+q c_{1} \sin \nu \int^{\tau} u d \tau^{\prime}=0
$$

in normalized form, with some real coefficients $c_{1}, q$, and $\nu$ depending on the $\Omega_{j}$, $\left|\mu_{j}\right|$, and initial values of $w_{j}$. Equation (11) is a generalized double sine-Gordon equation. Under certain conditions, it becomes precisely the so-called double sine-Gordon equation, when the argument of the second sine function is the second-harmonic of argument of the first one $(\nu=2)$. Both original and generalized double sG equations admit breather solutions, examples of which are shown on Fig. 4.

\subsection{Circular polarization - long wave case}

Importantly, both mKdV and $\mathrm{sG}$ models can be generalized in order to incorporate wave polarization dynamics. In the case of the mKdV model, i.e., when only the UV 


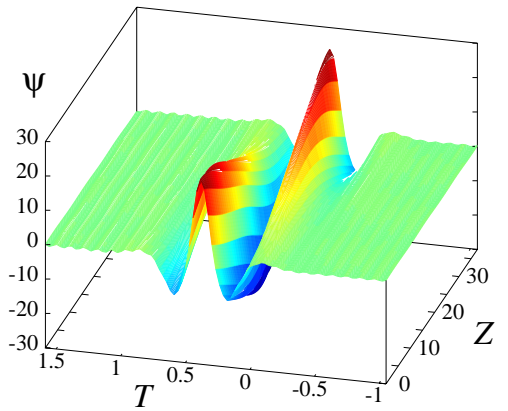

(a)

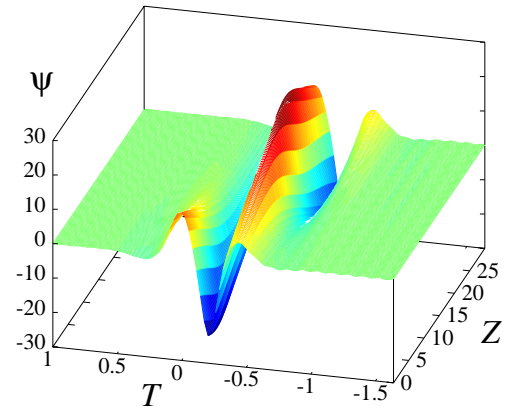

(b)

Fig. 4. Breathers of the generalized double sG equation. Left: $\nu=2, q=0.2$; right: $\nu=\sqrt{3}$, $q=0.4$.

transition is considered, assuming as above that the wave central frequency is very far from the resonance, the normalized vector electric field $(U, V)$ obeys the following system of two coupled mKdV equations [20]:

$$
\begin{aligned}
& U_{Z}=U_{T T T}+\left[\left(U^{2}+V^{2}\right) U\right]_{T}, \\
& V_{Z}=V_{T T T}+\left[\left(U^{2}+V^{2}\right) V\right]_{T} .
\end{aligned}
$$

Setting

$$
f=U+i V,
$$

the coupled mKdV equations Eqs. (12) and (13) reduce to

$$
f_{Z}=f_{T T T}+\left(|f|^{2} f\right)_{T},
$$

which is the complex modified Korteweg-de Vries (cmKdV) equation. However, confusion must be avoided between equation (15) and the other cmKdV equation

$$
f_{Z}=f_{T T T}+\left(|f|^{2}\right) f_{T}
$$

Indeed, Eq. (16) is completely integrable [21] while Eq. (15) is not. Equation (15) is usually referred to as cmKdV I, while Eq. (16) is called cmKdV II.

Linearly polarized solitons can be described by the solutions of Eq. (15) of the form

$$
f=f_{0}(Z, T) e^{i \phi},
$$

where $f_{0}$ is a solution of the real $\mathrm{mKdV}$ equation and $\phi$ is a constant.

More interesting would be a circularly polarized soliton, of the form

$$
f=u(T-w Z) e^{i(\omega T-k Z)} .
$$

However no exact, even numerical, steady state solution of this type do exist. It is however shown that very robust approximate ones do exist. An example is shown on Fig. 5 .

The circularly polarized FCP is unstable in the sub-cycle range (for $\omega \tau_{p}<1.4$ ), as appears on Fig. 6. The initial circularly polarized sub-cycle pulse evolves into a single-humped, linearly polarized pulse, which is nothing but a fundamental soliton of the real $\mathrm{mKdV}$ equation. 


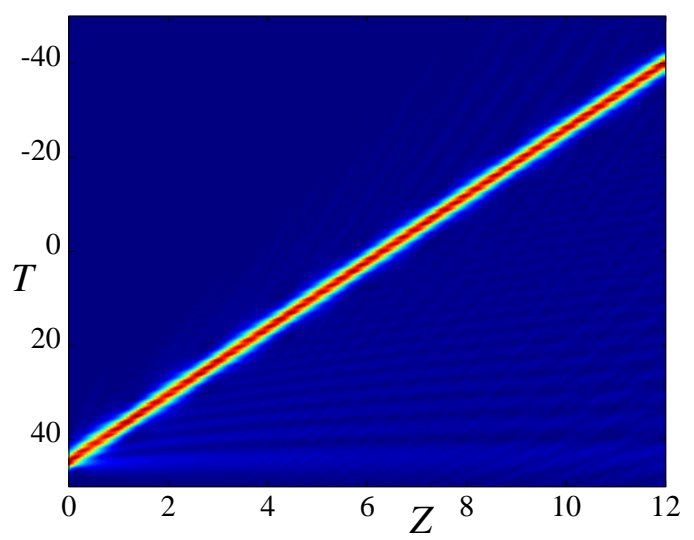

Fig. 5. The stable circularly polarized FCP: amplitude $|u|$.

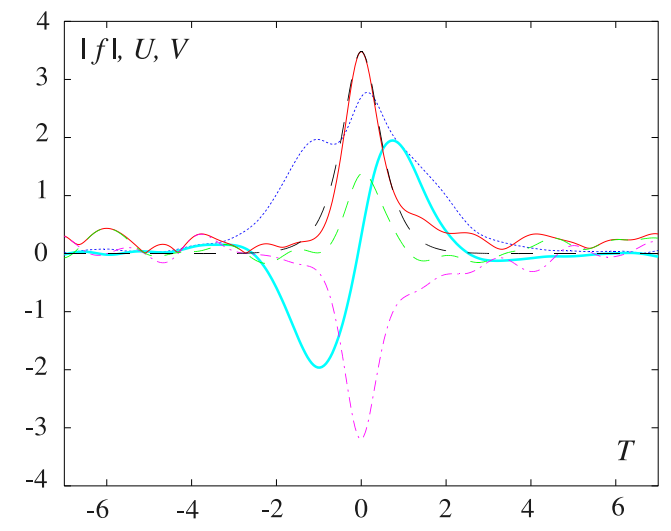

Fig. 6. The instability of the circularly polarized FCP in the sub-cycle range. Initial $(Z=$ 100): breather with $\omega=1, \tau_{p}=1$. Blue (dotted line), $|f|$; light blue (thick solid line), $U=\operatorname{Re}(f)$. Final $(Z=10000)$ : Red (solid line), $|f|$; pink (dash-dotted line): final, $U$; green (short dashing): final, $V=\operatorname{Im}(f)$. Black (long dashed line): fit of final $|f|$ by a fundamental soliton of the real mKdV model.

\subsection{Circular polarization - short wave case}

The case of one IR transition, i.e., when we intend to generalize the sG model to a vectorial normalized field $(U, V)$, is far from being identical, although analogous features are observed. Since the excited state for $x$-polarization cannot be identified with the excited state for $y$-polarization, two distinct population differences $w_{1}$ and $w_{2}$ are required. The asymptotic equations cannot be reduced to coupled sG-type equations, but yield a complicated system instead, which can be written in complex form as [22]:

$$
\begin{aligned}
f_{Z} & =-P_{T}, \\
P_{T} & =-M-i U K, \\
M_{T} & =w f-s f^{*}, \\
K_{T} & =\operatorname{Im}\left(f^{*} P\right), \\
s_{T} & =f M, \\
w_{T} & =-3 \operatorname{Re}\left(f^{*} M\right),
\end{aligned}
$$




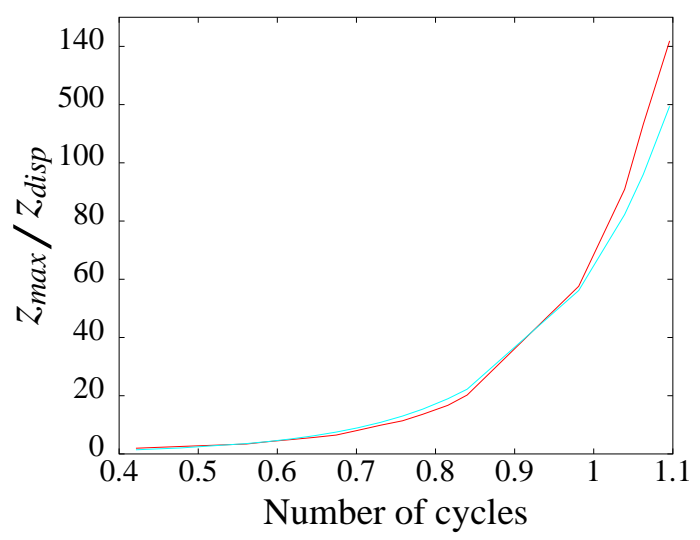

Fig. 7. The lifetime of the circularly polarized FCP, measured in units of the dispersion length, against the number of optical cycles in the pulse (Red line: numerical data. Light blue line: exponential fit).

where $f$ is the complex electric field defined as in Eq. (14) above, $P=P_{x}+i P_{y}$ is the polarization density, $w=\left(w_{1}+w_{2}\right) / 2, s=\left(w_{2}-w_{1}\right) / 2-i S$ are the population differences ( $S$ is the coherence between the two excited states), and the real quantity $K$ and the complex one $M$ are auxiliary fields.

As in the case of the cmKdV model, we can retrieve the scalar model form for linear polarizations by setting $f$ as in Eq. (17); then the system (19)-(24) reduces to the sG equation. More interesting are the solutions corresponding to circularly polarized FCP solitons. As in the case of the cmKdV, it is seen that no exact solutions of this type exist, whereas robust approximate ones do exist.

An approximate solution valid in the SVEA can be derived, as

$$
f=b e^{i\left[\left(k-\frac{b^{2} k}{\omega^{2}}\right) Z-\omega T\right]} \operatorname{sech}\left[b\left(T+\frac{k}{\omega} Z\right)\right] .
$$

The solution given by (25) can be used as initial data for the numerical resolution of the system (19)-(24). It is shown that the circular pulse is stable in the SVEA limit, but it is unstable in the sub-cycle range. Figure 8 shows the initial circular polarization, and the final state with two pulses having orthogonal linear polarizations.

\section{Supercontinuum generation}

\subsection{Supercontinuum generation in the $\mathrm{mKdV}$ and $\mathrm{sG}$ models}

It is known that an intense laser pulse launched in an optical fiber experiences spectral broadening, which can extend to several octaves, generating white light flashes, whose coherence largely depends on the input pulse parameters [23-26]. Experiments have been performed in particular in photonic crystal fibers and in tapered fibers, where confinement and nonlinearity can be exacerbated. A GNLSE model is used in general to confront the experimental data. However, the GNLSE model requires numerous parameters, whereas fundamentally, it remains based on the SVEA, whose validity is restricted to less than one octave spectral width. In spite of the good agreement between some theoretical works using GNLSE and experimental results, the principle 


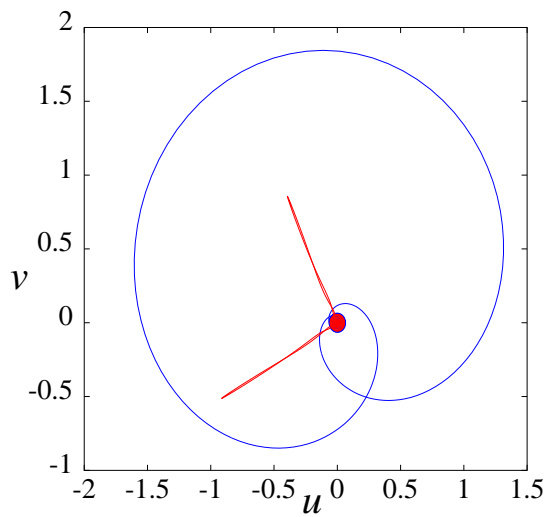

Fig. 8. Trajectory of the electric field vector $(u, v)=(\operatorname{Re}(f), \operatorname{Im}(f))$ in the transverse plane of the wave. Blue: initial, one circularly polarized pulse, red: final, two linearly polarized pulses.

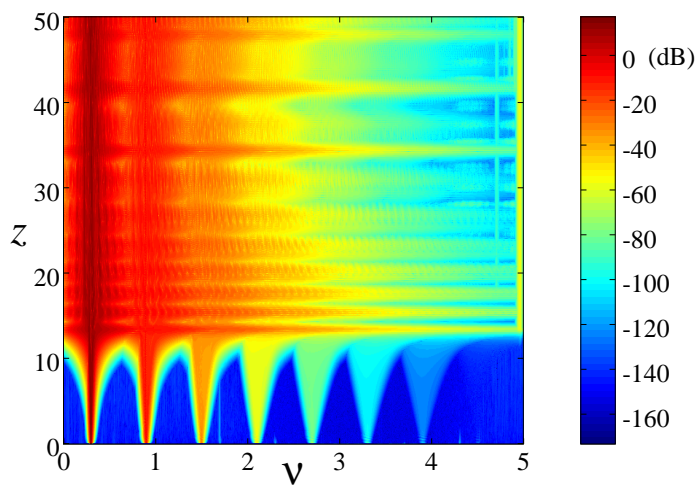

Fig. 9. Supercontinuum spectrum generation through dynamics modeled through mKdV equation, input with $F W H M=100, \nu=0.3$, and $A=0.7$.

of a SVEA is highly questionable from the fundamental point of view. We show in the present paper that the non-SVEA models presented above are consistent with supercontinuum generation with a reduced number of model parameters.

The mKdV equation (4) with $c_{1}=0$, in a normalized (dimensionless) form with $c_{2}=c_{3}=1$, is solved numerically using a standard fourth-order Runge-Kutta scheme, and finite differences for the $t$-derivatives. The initial condition is a Gaussian pulse, with carrier angular frequency $\omega$ and duration $\tau$ :

$$
u(0, t)=A \sin (\omega t) e^{-t^{2} / \tau^{2}}
$$

The ratio between the optical period $2 \pi / \omega$ and the pulse duration $\tau$ is chosen to be the same as in a $100 \mathrm{fs}$ long pulse with wavelength $\lambda=1 \mu \mathrm{m}$. Therefore, it is more convenient to do as if $t$ was expressed in fs and $\omega=2 \pi c / \lambda$, with $c=0.3 \mu \mathrm{m} / \mathrm{fs}$, and $\tau=F W H M / \sqrt{2 \ln 2}$, where $F W H M$ is the full width at half maximum of the pulse. Numerical resolution shows that a very broad spectrum quickly develops, see Fig. 9. 


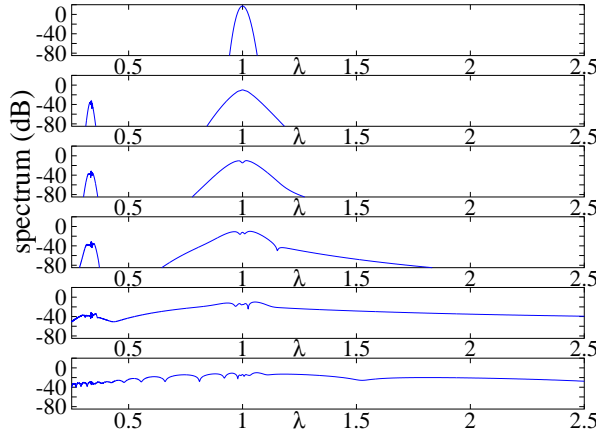

(a)

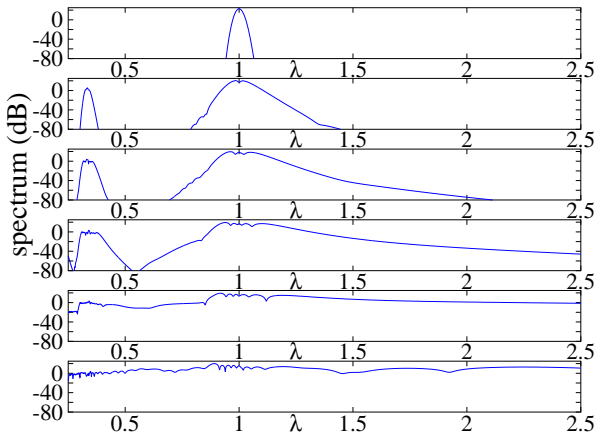

(b)

Fig. 10. a: The mKdV model, input with $F W H M=100, \nu=0.3, A=0.7$, for $z=0,3.1$, $6.2,9.3,12.4$, and 15.5. b: The sG model, input with $F W H M=100, \nu=0.3, A=2.5$, for $z=0,0.8,1.6,2.4,3.2$, and 4 .

\subsection{Generation of long wavelengths}

Among the questions which naturally arise, let us first ask ourselves how frequencies lower than the initial pulse frequency $\omega$ can be generated? The low-frequency spectrum is more easily seen when the spectrum is plotted against the wavelength $\lambda$. Figure 10(a) shows this spectrum for the mKdV model, whereas Fig. 10(b) is the analogous one for the sG model, starting with the same input (26) as above, corresponding to a 100 fs pulse, $\lambda=1 \mu \mathrm{m}$. By comparing Fig. 10(a) to Fig. 10(b) it is seen that analogous spectrum profiles are obtained when either the pulse duration or its amplitude is varied. In the case of the $\mathrm{mKdV}$ model, a few specific features are observed, see Fig. 10(a). A supercontinuum is generated in both cases; there is a strong asymmetry between Stokes and anti-Stokes sides. The asymmetry is more pronounced in the case of the sG model (Fig. 10(b)), which in addition shows more broadening towards large wavelengths than the mKdV model (Fig. 10(a)), for which the spectrum first extends towards the short wavelength, the anti-Stokes side.

Recalling that the mKdV model accounts for the UV transitions, while the sG model accounts for the IR transitions, a complete description of a general medium naturally requires both models. The extension of the spectrum towards low frequencies at the beginning of the supercontinuum generation process is frequently attributed to the effect of Raman scattering, where Raman scattering is due to the interaction of light with the IR transitions of the medium. The spectral broadening towards low frequencies we observe is thus nothing but the one due to Raman scattering. Hence, although we cannot pretend that the sG model describes the Raman effect alone, we see that it gives an account of the corresponding spectral broadening.

\subsection{Self-phase modulation}

Let us consider a quasi-monochromatic wave with angular frequency $\omega$ and wave vector $k$, as

$$
u=U(z, t) e^{i(k z-\omega t)}+c c+u_{1}(z, t)
$$

where $U$ is the amplitude of the fundamental wave, $c c$ holds for 'complex conjugate', and $u_{1}$ is some small correction. Equation (27) is reported into Eq. (4), in which we 


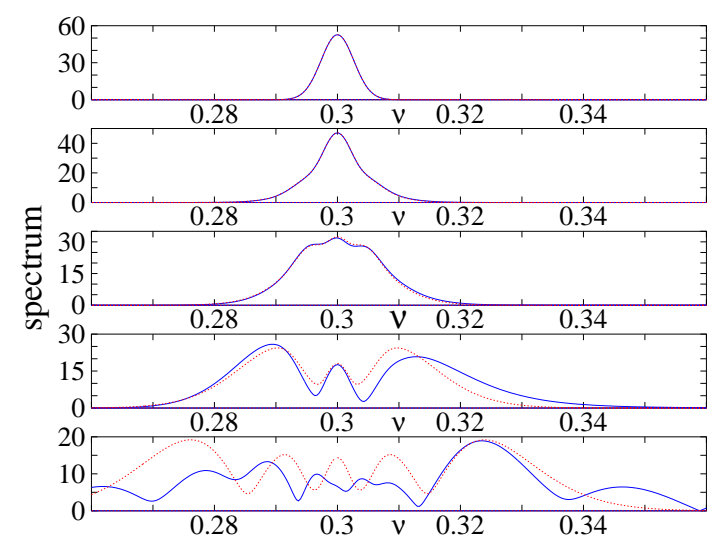

Fig. 11. Analytical self-phase modulation effect (red dotted lines), vs the mKdV model (blue solid lines); input with $F W H M=100, \nu=0.3, A=0.7$, and for $z=0,2,4,10$, and 20 .

neglect dispersion, and disregard third harmonic generation, which yields

$$
U=\frac{A}{2} \exp \left[i\left(\frac{c_{1}}{2 \omega^{3}}+3 \omega c_{2}\right) \frac{A^{2}}{4} z\right] .
$$

Equation (28) accounts for the self-phase modulation (SPM), a well-known phenomenon which leads to a broadening of the spectrum with typical oscillations of the spectral density. We can compare the analytical expression of the self-phase modulation given by Eq. (28) to the numerical solution of the mKdV equation, see Fig. 11. It is seen that the analytic envelope approximation coincides with numerics until $z \simeq 4$, then actual broadening becomes asymmetric, while analytic formula remains symmetric. This shows that the very beginning of the broadening process is actually due to self-phase modulation.

\subsection{High harmonics generation}

Lots of high harmonics are created and involved in the process of supercontinuum generation. Such effect cannot be accounted for within the SVEA unless an amplitude is explicitly included for each harmonic in the model. Up to 15 harmonics can be seen in the example of Fig. 12. Oscillations of their amplitudes can be observed, and are easily retrieved by a simple analytical approach.

We can gain further understanding from a simple analytic approach of the third harmonic generation. Let us indeed consider the approximate solution of the mKdVsG equation given by (27) and (28). The correction term $u_{1}$ in (27) is a third harmonic

$$
u_{1}(z, t)=V(z, t) e^{i\left(k^{\prime} z-3 \omega t\right)}+c c+u_{2}(z, t),
$$

with $k^{\prime}=k(3 \omega)$ and $u_{2}$ a higher-order correction term. Its amplitude $V$ can be computed with the same approximations as above, as

$$
\frac{\partial V}{\partial z}=i\left(\frac{-c_{1}}{6 \omega^{3}}+3 \omega c_{2}\right) U^{3} e^{i\left(3 k-k^{\prime}\right) z} .
$$

Assuming that $U$ is a constant, and no incident third harmonic $(V=0$ at $z=0)$, this expression leads to well-known oscillations of the third harmonic amplitude due to 


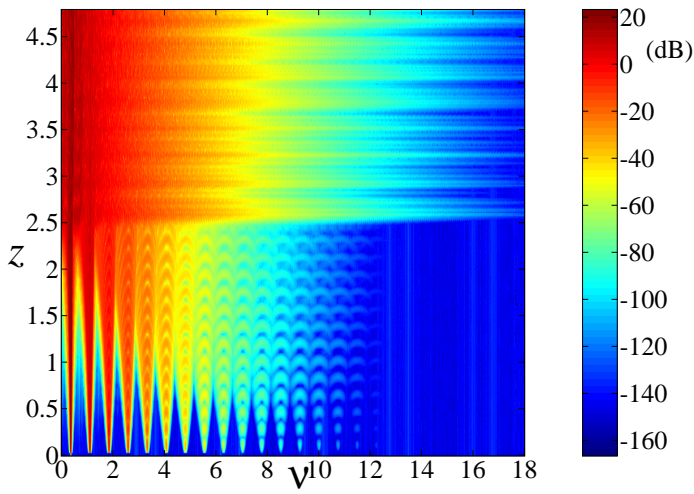

Fig. 12. Initial pulse with $F W H M=80, \nu=0.375$, and $A=5$, according to the sG model. Notice the high number of harmonics, and the oscillations of their amplitudes.

the phase mismatch $\Delta k=3 k-k^{\prime}$, with $V \propto \sin \frac{\Delta k z}{2}$. Notice that, if the SPM effect is important, the phase of $U$ in Eq. (30) evolves according to Eq. (28), and consequently the phase mismatch $\Delta k$ should be replaced by

$$
\Delta k^{\prime}=3 k-k^{\prime}+3\left(\frac{c_{1}}{2 \omega^{3}}+3 \omega c_{2}\right) .
$$

These simple analytical results are found to be in quantitative agreement with numerics. Let us now determine the spectral width of harmonics. Assuming a Gaussian profile

$$
u=A e^{-\frac{t^{2}}{\tau^{2}}} e^{-i \omega_{0} t}
$$

its Fourier transform has the well-known expression

$$
\hat{u}=\frac{2 \sqrt{\pi}}{A \tau} e^{-\frac{\left(\omega-\omega_{0}\right)^{2} \tau^{2}}{4}} .
$$

The $n$th harmonic evolves essentially as $u^{n}$ does, and is straightforwardly seen that its duration is $\tau / \sqrt{n}$. Consequently its Fourier transform is given by replacing $\tau$ by $\tau / \sqrt{n}$ (and $A$ by $A^{n}$ ) in (33), and hence the spectral width of the $n$th harmonic is

$$
\frac{2 \sqrt{n}}{\tau}
$$

It increases as $\sqrt{n}$. Figure 13 shows the comparison between the actual spectrum obtained by resolution of the mKdV equation (blue), and Gaussian profiles with width increasing as $\sqrt{n}$ (red). The agreement allows to conclude that the spectral width of the harmonics is actually governed by this law, at least at the considered stage of the process.

We expect also some spectral broadening due to parametric interaction between the sidebands of the harmonics and the ones of the fundamental. We denote by $\omega$ the fundamental angular frequency, and assume some sideband $\omega+\delta \omega$. Then the third harmonics contains the sideband $3(\omega+\delta \omega)$. It may interact with the fundamental with frequency $\pm \omega$, according to $3(\omega+\delta \omega)-\omega-\omega=\omega+3 \delta \omega$. Such a parametric process relates a sideband at $\omega+\delta \omega$ to another one at $\omega+3 \delta \omega$, and is expected to result in spectral broadening. However, it was difficult to get convincing numerical evidence of the occurring of this process, due to the concurrence of another one, which will be discussed in the next section. 


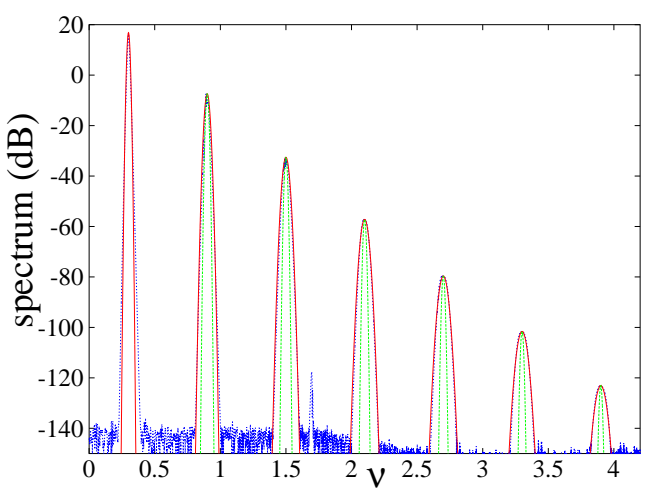

Fig. 13. Blue: $m K d V$ equation, Red solid lines: Gaussian with width increasing as $\sqrt{n}$, Green dotted lines: Gaussian with constant width. Here $F W H M=100, \nu=0.3$, and $A=0.7$, for $z=2$.

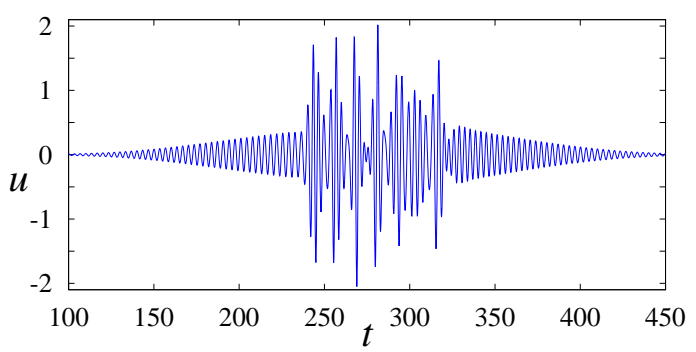

Fig. 14. Evolution of the profile of an initial pulse with $F W H M=100, \nu=0.3$, and $A=1$, according to the $\mathrm{sG}$ model at the end of our computation $(z=20)$.

\section{Few cycle solitons in supercontinuum generation}

\subsection{The sG model}

We solve numerically the sG equation, Eq. (4) with $c_{2}=c_{3}=0$, and $c_{1}=50$ (a change in $c_{1}$ results only in a change of the unit along the $z$-axis), with an initial data in a form of a Gaussian pulse as Eq. (26). For moderate amplitudes and high frequencies, a few FCP solitons form and tend to separate (Fig. 14).

However, due to the dispersion properties of the sG equation and the characteristics of the input pulse, their velocity is quite small; they don't separate completely, but interact forming some interference pattern, see panel (a) in Fig. 15. Nonlinear interaction between FCP solitons produces very sharp peaks, which induce a huge broadening of the spectrum, see see panel (b) in Fig. 15. Finally, the evolution of the supercontinuum generation process according to the sG model can be summarized as illustrated by Fig. 16. The input spectrum $(z=0)$ is the blue curve. First, it occurs the generation of harmonics (dark green, $z=4$ ), then the harmonics start to broaden (red, $z=8$ ), and the gaps between them start to fill. When the first soliton becomes apparent (light blue, $z=9.2$ ), the gaps are completely filled. Then the slope of the decay of the spectrum reduces, as seen in the green curve when the second soliton is just formed ( $z=11.16$ ), to reach eventually an ultrabroad spectrum (magenta, $z=20)$. 


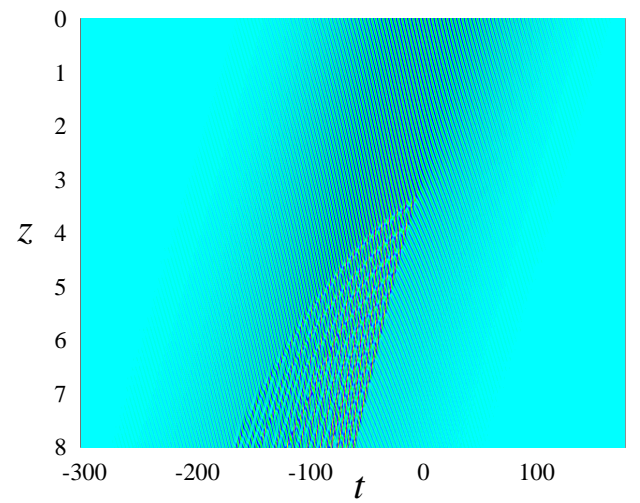

(a)

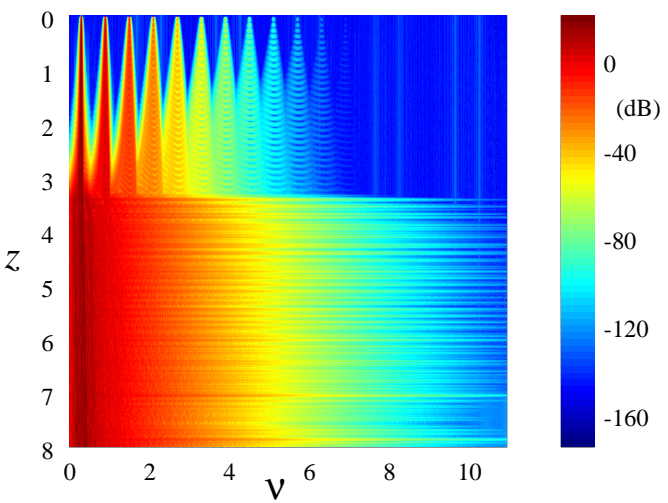

(b)

Fig. 15. Initial pulse with $F W H M=100, \nu=0.3$, and $A=2.5$, according to the sG model. a) FCP solitons form, don't separate, but interact; b) generation of an ultrabroad supercontinuum.

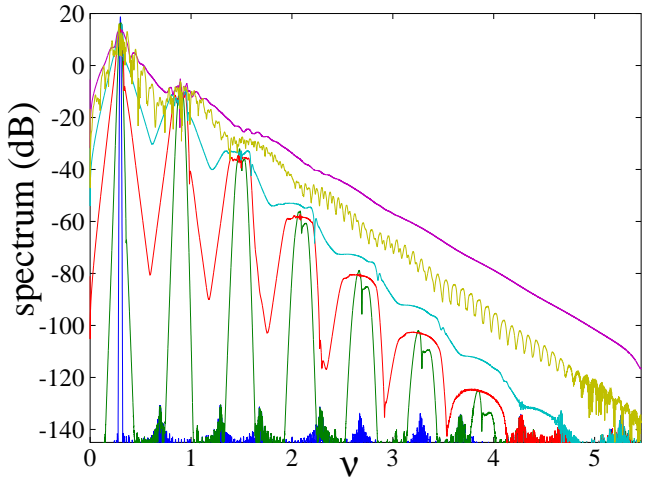

Fig. 16. Initial pulse with $F W H M=100, \nu=0.3$, and $A=1$, according to the sG model (logarithmic scale): blue $(z=0)$, dark green $(z=4)$, red $(z=8)$, light blue $(z=9.2)$, green $(z=11.16)$, magenta $(z=20)$. 


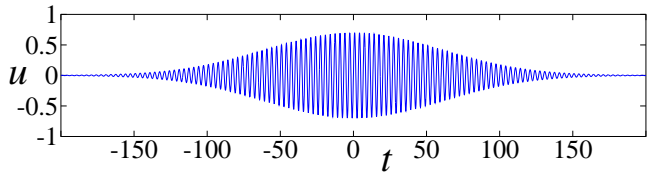

(a)

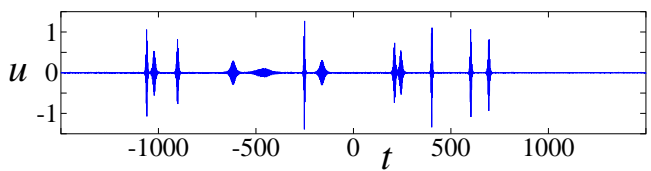

(b)

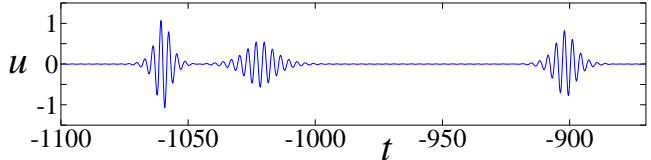

(c)

Fig. 17. Evolution of an initial pulse with $F W H M=100, \nu=0.3$, and $A=0.7$, according to the mKdV model (same as in Fig. 18). a) initial pulse, $z=0$, b) set of emerging FCP solitons, $z=999$, c) zoom on some FCP solitons in b) (the soliton speed is compensated for clarity).

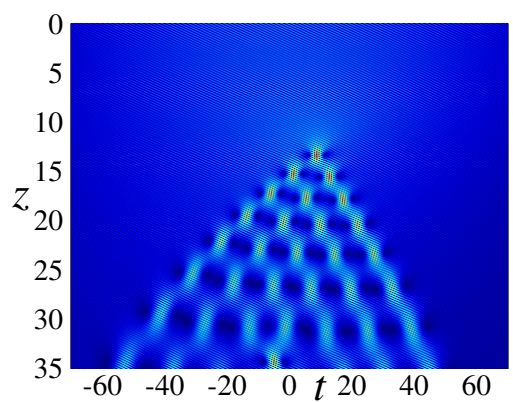

(a)

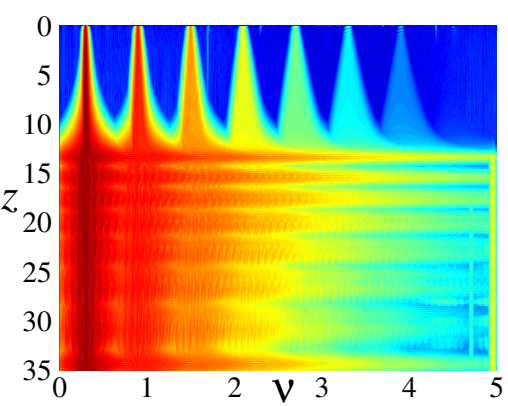

(b)

Fig. 18. Initial pulse with $F W H M=100, \nu=0.3$, and $A=0.7$, according to the mKdV model. left: temporal evolution, right: spectrum evolution.

\subsection{The mKdV model}

We solve in a same way the $\mathrm{mKdV}$ equation (4) with $c_{1}=0$, starting from the same initial data with $F W H M=100$ and $\nu=0.3$. At high amplitude level $(A \sim 2)$, an initial 100 fs pulse splits into a set of FCP solitons, which are mKdV breathers (Fig. 17). The FCPs form in a first stage until $z \simeq 12$ then slowly go away one from the other. A huge spectral broadening occurs when they form. Comparison between temporal profile and spectrum shows indeed that the spectrum extends at the very point when the solitons separate (Fig. 18). At a lower amplitude $(A \sim 0.1,0.2)$, it may happen that only two solitons form. They interact and form a kind of superbreather (Fig. 19). In the meantime, the spectrum oscillates, showing a recurrence to the initial state that recalls the Fermi-Pasta-Ulam recurrent dynamics (Fig. 20), see Ref. [27]. 


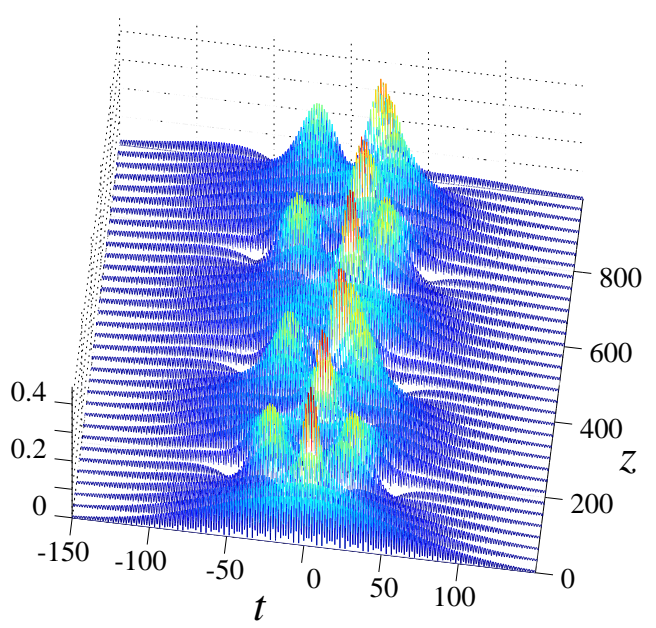

Fig. 19. Initial pulse with $F W H M=100, \nu=0.3$, and $A=0.17$. Two FCP solitons are formed, and propagate together as a higher order NLS 'super-breather'.

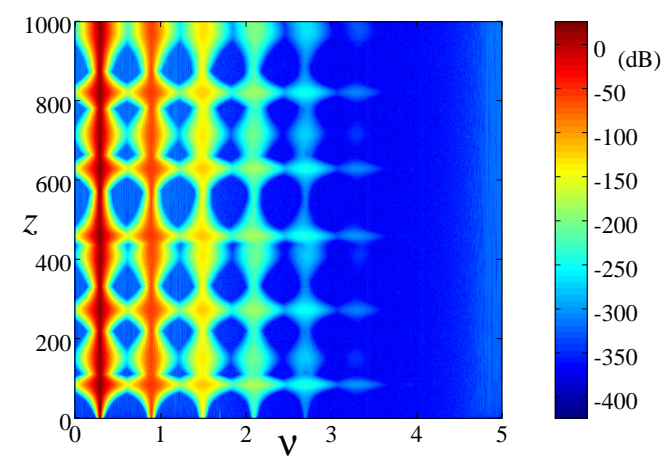

Fig. 20. Evolution of the 'super-breather' spectrum generated by an initial pulse with $F W H M=100, \nu=0.3$, and $A=0.17$.

\section{Conclusion}

We have presented three generic non-SVEA models: modified Korteweg-de Vries, sineGordon, and $\mathrm{mKdV}$-sG models. Initially developed in the frame of a scalar model, of a two-component medium in which each component obeys a two-level model, these models have been generalized to more complicated Hamitonians, and to vector fields. We showed numerically that circularly polarized few-cycle solitons are particularly robust, but when sub-cycle duration is considered, for a spectral bandwidth spanning over several octaves, these solitons become unstable and decay into one or two linearly polarized single-humped solitons.

The non-SVEA models numerically predict supercontinuum generation over several octaves, and we identified several mechanisms in the process. First, the sG term accounts for Raman broadening. Second, a modulation instability mechanism entails spectral broadening at the beginning of the process. Generation of high-harmonics has also been evidenced, with its relation to the formation of the supercontinuum. Finally, we observed that FCP solitons are formed in the process, and interact nonlinearly together: their formation is closely related to that of the ultrabroad spectrum, in correspondence with the known impact of picosecond/subpicosecond soliton for- 
mation for supercontinuum generation from GNLSE modeling. At present time, a definite advantage of non-SVEA models is that they do not require a high number of terms and coefficients, while they procure the possibility to explore few-cycle to sub-cycle ultrashort pulse dynamics on safer theoretical grounds. However, the tuning of non-SVEA models and parameters to realistic material properties and actual experiments calls for further studies.

\section{References}

1. R. Ell, U. Morgner, F.X. Kärtner, J.G. Fujimoto, E.P. Ippen, V. Scheuer, G. Angelow, T. Tschudi, M.J. Lederer, A. Boiko, B. Luther-Davies, Opt. Lett. 26, 373 (2001)

2. T. Brabec, F. Krausz, Phys. Rev. Lett. 78, 3282 (1997)

3. J.M. Dudley, G. Genty, S. Coen, Rev. Mod. Phys. 78, 1135 (2006)

4. J.S. Russell, 14th Meeting of the British Association for the Advancement of Science (1844)

5. J. Boussinesq, J. de Mathématiques Pures et Appliquées Sr. II 17, 55 (1872)

6. D.J. Korteweg, G. de Vries, Phil. Magazine 39 (240), 422 (1895)

7. I.V. Melnikov, D. Mihalache, F. Moldoveanu, N.-C. Panoiu, Phys. Rev. A 56, 1569 (1997)

8. H. Leblond, F. Sanchez, Phys. Rev. A 67, 013804 (2003)

9. H. Leblond, D. Mihalache, Phys. Rep. 523, 61 (2013)

10. H. Leblond, H. Triki, D. Mihalache, Rom. Rep. Phys. 65, 925 (2013)

11. D.J. Frantzeskakis, H. Leblond, D. Mihalache, Rom. J. Phys. 59, 767 (2014)

12. H. Leblond, J. Phys. B: At. Mol. Opt. Phys. 41, 043001 (2008)

13. M. Wadati, J. Phys. Soc. Jpn. 34, 1289 (1973)

14. R.K. Dodd, J.C. Eilbeck, J.D. Gibbon, H.C. Morris, Solitons and Nonlinear Wave Equations (Academic, London 1982)

15. H. Leblond, S.V. Sazonov, I.V. Mel'nikov, D. Mihalache, F. Sanchez, Phys. Rev. A 74, 063815 (2006)

16. M.J. Ablowitz, H. Segur, Solitons and the Inverse Scattering Transform (SIAM, Philadelphia, 1981)

17. K. Konno, W. Kameyama, H. Sanuki, J. Phys. Soc. Japan 37, 171 (1974)

18. H. Triki, H. Leblond, D. Mihalache, Opt. Comm. 285, 3179 (2012)

19. H. Triki, H. Leblond, D. Mihalache, Phys. Rev. A 86, 063825 (2012)

20. H. Leblond, H. Triki, F. Sanchez, D. Mihalache, Phys. Rev. A 83, 063802 (2011)

21. M.V. Foursov, J. Math. Phys. 41, 6173 (2000)

22. H. Leblond, H. Triki, D. Mihalache, Phys. Rev. A 84, 023833 (2011)

23. J.K. Ranka, R.S. Windeler, A.J. Stentz, Opt. Lett. 25, 2 (2000)

24. T.A. Birks, W.J. Wadsworth, P.St.J. Russell, Opt. Lett. 25, 1415 (2000)

25. J.H.V. Price, W. Belardi, T.M. Monro, A. Malinowski, A. Piper, D.J. Richardson, Opt. Express 10, 382 (2002)

26. U. Möller et al., Opt. Fiber Technol. 18, 304 (2012)

27. H. Leblond, Ph. Grelu, D. Mihalache, Phys. Rev. A 90, 053816 (2014) 